\title{
Tecnologias tangíveis e vestíveis como recursos para ambiente inclusivo: uma revisão sistemática
}

\author{
Eliana Alves Moreira ${ }^{1,2}$, M. Cecília C. Baranauskas ${ }^{1}$ \\ ${ }^{1}$ Instituto de Computação - Universidade Estadual de Campinas (UNICAMP) \\ Av. Albert Einstein, 1251 - 13.083-852 - Campinas - SP - Brasil \\ ${ }^{2}$ Universidade Federal de Itajubá (UNIFEI) \\ Av. BPS, 1303 - 37.500-903 - Itajubá - MG - Brasil \\ \{eliana.moreira, cecilia\}@ic.unicamp.br
}

\begin{abstract}
Digital technologies have been used as mediators for the teachinglearning process in inclusive environments. However, little is known about the potential use of tangible and wearable contemporary technologies in this scenario. In this work we present a systematic review of studies published between 2000 and 2015, in the field of contemporary technologies as tools for inclusive school environments. Results indicate that most of the studies are based on tangible interface or touch-based interaction, and that wearable technologies and gesture-based interactions are still largely unexplored, suggesting new fields of research.
\end{abstract}

Resumo. Tecnologias digitais vêm sendo utilizadas como mediadoras do processo ensino-aprendizagem em ambientes inclusivos. Porém, pouco se sabe sobre o potencial de uso de tecnologias contemporâneas tangiveis e vestiveis nesse cenário. Neste trabalho realizamos uma revisão sistemática de estudos publicados entre 2000 e 2015, na área de tecnologias contemporâneas como ferramentas para ambientes escolares inclusivos. Resultados indicam que a maioria dos estudos é baseada em interface tangível ou interação por toque e que tecnologias vestíveis e interações gestuais ainda são pouco exploradas, sugerindo novos campos de pesquisa.

\section{Introdução}

Vários recursos auxiliam o processo de aprendizado em ambientes escolares inclusivos, tais como materiais didáticos e pedagógicos acessíveis, tecnologias de informação e de comunicação (TICs) acessíveis, recursos ópticos, entre outros [Ropoli et al. 2010].

Neste trabalho argumentamos que TICs têm potencial para desempenhar um importante papel na promoção da inclusão, aprimorando a utilização dos materiais pedagógicos e acrescentando novos recursos no âmbito sócio-educacional, pois, como afirmam Galvão Filho e Damasceno (2008), a presença crescente das TICs aponta para diferentes formas de relacionamento com o conhecimento e sua construção, assim como novas concepções e possibilidades pedagógicas. $\mathrm{O}$ uso da informática aliado à constante renovação de equipamentos e softwares especialmente desenvolvidos serve como mediador no processo de ensino-aprendizagem nos mais diversos campos do conhecimento [Araújo, Brito e Silva 2013]. 
Neste contexto, diversas soluções tecnológicas vêm sendo utilizadas como recursos inclusivos; porém, tecnologias contemporâneas, dentre as quais podemos destacar aquelas que apresentam interface tangível com o usuário, têm se destacado especialmente em cenários de pesquisa acadêmica. As interfaces tangíveis dão forma física à informação digital, empregando artefatos físicos que quando manipulados funcionam como representações e controles para mídia computacional, proporcionando uma resposta ou retorno ao usuário [Ullmer e Ishii 2000].

Embora estas tecnologias contemporâneas tenham o seu potencial de uso como recursos inclusivos reconhecido, pouco se sabe, da literatura e de práticas escolares, sobre como ferramentas que utilizam estas tecnologias estão sendo propostas em cenários nacional e internacional e para quais audiências estariam sendo propostas. Além disso, revisões encontradas na literatura não tratam especificamente da utilização destas ferramentas contemporâneas em ambientes escolares inclusivos: por exemplo, entre outros trabalhos, Sheu e Chen (2014) relatam sobre ferramentas de interação gestual para ambientes escolares não inclusivos e outros autores tratam de ferramentas para características específicas, como o autismo, tratado por Khowaja e Salim (2013).

Assim, este trabalho apresenta uma revisão sistemática da literatura (RSL) para sintetizar e avaliar como pesquisadores estão propondo o uso de tecnologias contemporâneas, principalmente as tangíveis e as vestíveis, como recursos a serem utilizados em ambientes escolares inclusivos. A RSL incluiu estudos publicados de 2000 a 2015 nos principais veículos acadêmicos nacionais e internacionais da área de Informática e Educação. A estratégia utilizada foi a busca automática em sete fontes de publicações e a busca manual pelos trabalhos citados como "trabalhos relacionados" nos estudos incluídos na revisão. A busca automática retornou 864 publicações. A busca manual foi feita para 33 artigos. Ao todo, após eliminação de duplicatas e seguindo critérios de exclusão e inclusão, 46 estudos $^{1}$ tiveram análises mais detalhadas, apresentadas neste trabalho, que está organizado da seguinte maneira: a próxima seção apresenta o método utilizado na RSL, seguida por uma visão geral dos estudos incluídos, a categorização e análise dos resultados encontrados e por fim, a conclusão.

\section{Método}

Esta seção apresenta os passos para a realização da RSL, que foi baseada na proposta da metodologia Prisma [Moher et al. 2009] de quatro fases: Identificação, Triagem, Elegibilidade e Incluídos.

\subsection{Questões de pesquisa}

O objetivo desta RSL é realizar um estudo para coletar conhecimento, sintetizar, avaliar e apresentar um panorama de como pesquisadores estão propondo recursos que utilizam como principal meio de interação tecnologias contemporâneas em ambientes escolares inclusivos. A questão principal de pesquisa é: "De que maneira as tecnologias contemporâneas, especialmente as vestiveis e tangíveis, estão sendo utilizadas como recursos em ambientes escolares inclusivos?". A partir da questão principal, outras três questões mais específicas foram definidas e estão descritas na Tabela 1.

\footnotetext{
1 A relação das publicações analisadas nesta RSL pode ser acessada em http://www.ic.unicamp.br/ ra120437/SBIE2015.
} 


\subsection{Fontes e Critérios de Seleção de Busca}

De acordo com as questões de pesquisa, a busca foi elaborada envolvendo tipo de tecnologia, ambiente escolar, abordagem ao design e acessibilidade dos recursos: (a) Tecnologia: tangible interface, TUI, tangible user interface, wearable technology, wearable computing; (b) Ambiente escolar: inclusive school, special education, inclusive education; (c) Design universal: universal design, design for all, technology for all, technology to all; (d) Acessibilidade: assistive technology, assistive resource.

Tabela 1 - Questões específicas de pesquisa

\begin{tabular}{|l|l|}
\hline \multicolumn{1}{|c|}{ Questão de Pesquisa } & \multicolumn{1}{c|}{ Motivação } \\
\hline $\begin{array}{l}\text { QP1 - Qual o tipo de tecnologia e } \\
\text { quais os dispositivos utilizados? }\end{array}$ & $\begin{array}{l}\text { Esta questão objetiva compreender como os pesquisadores estão criando diferentes } \\
\text { soluções com as tecnologias contemporâneas como recursos inclusivos. }\end{array}$ \\
\hline $\begin{array}{l}\text { QP2 - A solução foi desenvolvida } \\
\text { para um público específico? }\end{array}$ & $\begin{array}{l}\text { Esta questão objetiva compreender se os pesquisadores estão criando soluções } \\
\text { voltadas para um público específico ou soluções universais. }\end{array}$ \\
\hline $\begin{array}{l}\text { QP3 - A ferramenta foi testada? Se } \\
\text { sim, como e com quem? }\end{array}$ & $\begin{array}{l}\text { Esta questão visa verificar se a ferramenta já se caracteriza como um estudo prático } \\
\text { ou ainda somente uma proposta conceitual. Além disso, é importante saber se a } \\
\text { ferramenta já foi testada com usuários finais e no contexto ao qual será realmente } \\
\text { utilizada ou se somente foi testada em laboratório por especialistas. }\end{array}$ \\
\hline
\end{tabular}

De acordo com as questões de pesquisa, foram definidas sete de fontes de busca: ACM (Association for Computing Machinery); BJET (British Journal of Education Technology); CEIE (Comissão Especial de Informática na Educação); IEEE (Institute of Electrical and Electronics Engineers); JETS (Journal of Education Technology \& Society); Science Direct e Springer ${ }^{2}$.

Para a busca na fonte CEIE foi utilizada tanto string de busca em português, quanto em inglês. Palavras parecidas ou no plural foram tratadas de acordo com os critérios de busca da fonte, isto é, colocando caracteres coringa ou explicitamente, sendo este último quando o motor de busca da fonte não permitiu a utilização de caracteres coringa. Os termos de busca foram pesquisados em todos os campos disponíveis.

Apenas cinco destas fontes de busca aceitaram a string como foi desenvolvida. Para realizar as buscas no Portal de Publicações da CEIE e no Portal da IEEE, alterações tiveram que ser realizadas na string pré-definida. O Portal de Publicações da CEIE, cuja biblioteca armazena trabalhos de CBIE (Congresso Brasileiro de Informática na Educação), RBIE (Revista Brasileira de Informática na Educação), SBIE (Simpósio Brasileiro de Informática na Educação) e WIE (Workshop de Informática na Escola) somente aceita strings de busca menores que o tamanho daquela especificada neste trabalho. Desta forma, a busca nesse portal foi realizada utilizando-se as partes integrantes das cláusulas mais externas (separadas pelo "AND”). $\mathrm{O}$ Portal da IEEE também não aceitou a busca com a string com mais de 40 palavras. A solução, então, foi dividir as cláusulas, fazendo uma combinação entre elas, e refazer a busca com cada combinação. Foram geradas nove combinações ${ }^{3}$, cujos resultados são complementares e as duplicatas foram eliminadas na fase "Triagem". Quando pertinentes à pergunta de

\footnotetext{
2 ACM - http://dl.acm.org/advsearch.cfm?coll=DL\&dl=ACM\&query=; BJET - http://onlinelibrary.wiley.com; CEIE http://www.br-ie.org/pub/index.php/index/search/search; IEEE - http://ieeexplore.ieee.org; JETS - http://www.ifets.info; Science Direct - http://www.sciencedirect.com; Springer - http://link.springer.com

3 A string de busca original, a string de busca auxiliar (para a fonte CEIE) e as combinações (para a fonte IEEE) podem ser vistas em http://www.ic.unicamp.br/ ra120437/SBIE2015.
} 
pesquisa, trabalhos citados como "trabalhos relacionados" nos estudos incluídos nesta RSL foram buscados manualmente a fim de compor o rol de publicações a serem analisadas. Foram selecionados 33 trabalhos manualmente e, após análise, cinco deles foram escolhidos para constituir parte da RSL.

\subsection{Seleção de estudos}

Para evitar o risco de viés na RSL, foram definidos alguns critérios de exclusão e de inclusão, descritos na Tabela 2.

Tabela 2 - Critérios de exclusão e de inclusão de artigos no estudo

\begin{tabular}{|l|l|}
\hline Critérios de exclusão & Critérios de inclusão \\
\hline Estudos realizados antes de 2000 & Estudos que utilizam tecnologias contemporâneas, \\
Short-papers & tais como as vestíveis ou tangíveis \\
Estudos duplicados & Estudos cuja aplicação permite o ensino inclusivo \\
Estudos que não citam o autor ou a tecnologia utilizada & Estudos que apresentam uma ferramenta para \\
Estudos que não pertencem ao escopo deste trabalho & ensino inclusivo \\
Estudos em idiomas diferentes de inglês e português & \\
Livros, pôsteres, índices, abstracts, notas, comentários, Front matter & \\
\hline
\end{tabular}

Com estes critérios definidos, as buscas foram realizadas nas fontes descritas anteriormente, no dia 02 de abril de 2015. Foram identificados 864 estudos $^{4}$, dos quais foram excluídos manualmente 132 livros e/ou trabalhos realizados antes do ano 2000 (algumas fontes de busca não permitiam este filtro automaticamente), restando assim 732 estudos, os quais, juntamente com os artigos que foram buscados manualmente, compõem a fase "Identificação" do modelo Prisma. Na fase "Triagem" foram excluídos outros 89 estudos que se tratavam de duplicatas, comentários, índices, notas, pôsteres, abstracts, publicações sem autor, editoriais etc. Na fase "Elegibilidade" 585 artigos foram eliminados com base nos critérios de exclusão e inclusão, analisando-se primeiramente o título e o abstract. Aqueles artigos que passaram por este primeiro filtro foram analisados mais atenciosamente, a fim de verificar se estes realmente atendiam aos critérios de inclusão. $\mathrm{Na}$ fase "Incluídos" após análise dos estudos, verificou-se que alguns deles apresentavam a mesma ferramenta para ensino inclusivo, sob uma ótica diferente. Por este motivo, 13 estudos foram ainda excluídos, respeitando o critério de manter aquele que retratava a ferramenta de forma mais completa. Deste modo, finalmente, 46 estudos foram selecionados para fazerem parte desta revisão sistemática. Assim, com os estudos incluídos na revisão, foi feita a extração de dados.

\section{Resultados}

A Figura 1.a apresenta uma visão geral dos estudos incluídos na RSL. O primeiro estudo foi publicado em 2005, apesar de esta RSL considerar trabalhos desde 2000. O ano de 2015 apresenta somente um estudo, possivelmente devido ao fato de o processo de busca por publicações ter sido realizado no início do $4^{\circ}$. mês do ano de 2015 . Pode ser observado que existe um crescimento bastante relevante a partir de $2012^{5} \mathrm{em}$ publicações relativas ao tema desta RSL, o que reflete as oportunidades de estudos que as tecnologias contemporâneas podem proporcionar ao campo de ambientes inclusivos, especialmente pela difusão do uso destas tecnologias pelas pessoas.

\footnotetext{
4 A quantidade de resultados retornados de cada fonte de busca pode ser vista em http://www.ic.unicamp.br/ ra120437/SBIE2015.

${ }^{5}$ Neste ponto é importante salientar que os 13 estudos eliminados (por tratarem sobre uma mesma ferramenta que outros estudos) eram distribuídos da seguinte forma em relação ao ano de publicação (o número entre parênteses refere-se à quantidade de estudos eliminados no ano): 2007 (1); 2008 (1); 2009 (1); 2011 (3); 2012 (1); 2013 (5) e 2014 (1).
} 
Foram analisadas publicações provenientes de quatro continentes (Figura 1.b), sendo 23 países no total ${ }^{6}$. Estados Unidos é o país com mais publicações, $14,54 \%$, seguido por Espanha e Grã-Bretanha, com 10,91\% e 9,09\%, respectivamente. O Brasil aparece na sétima posição, com $5,45 \%$ das publicações. Porém, ao observarmos o continente de onde provêm as publicações, a Europa possui $41,82 \%$, seguida por América, Ásia e Oceania, com 36,36\%, 20\% e 1,82\%, respectivamente.

(a)

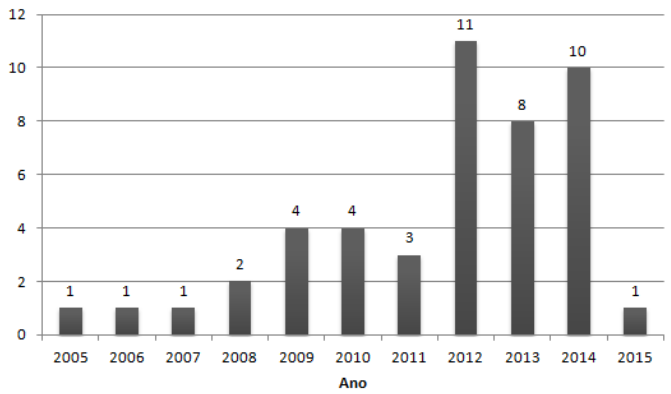

(b)

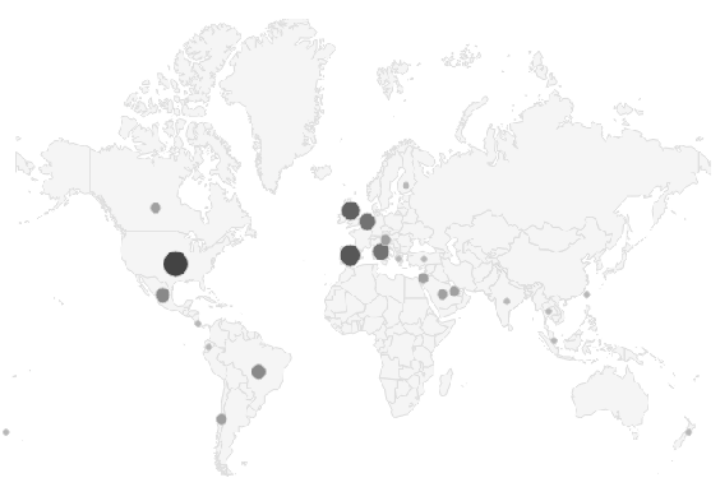

Figura 1 - (a) Publicações por ano; (b) Publicações por país

\subsection{Respondendo perguntas de pesquisa}

Como descrito na Seção 2, esta RSL tem foco em três questões de pesquisa. Assim, os dados foram extraídos baseando-se nestas questões.

A primeira pergunta específica (QP1) diz respeito a qual tipo de tecnologia e quais dispositivos são utilizados pelas ferramentas apresentadas nos estudos. A Figura 2.a mostra que quase metade das ferramentas utilizam interface tangível e 30,43\% usam o toque como forma de interação com o usuário. Apenas uma ferramenta utiliza somente tecnologia vestível, a qual consiste no uso de i-glasses, que sobrepõem imagens virtuais na visão do mundo real. A Figura 2.b mostra a quantidade de estudos que utilizam os determinados tipos de interação/interface, uma vez que alguns deles utilizam mais de um tipo na ferramenta. Das 30 interfaces tangíveis, nove delas são realizadas via objetos dispostos em tabletop, representando $30 \%$ do total de dispositivos utilizados pela interface tangível. As interfaces gestuais foram utilizadas em estudos dos anos 2011, 2012 e 2013.

(a)

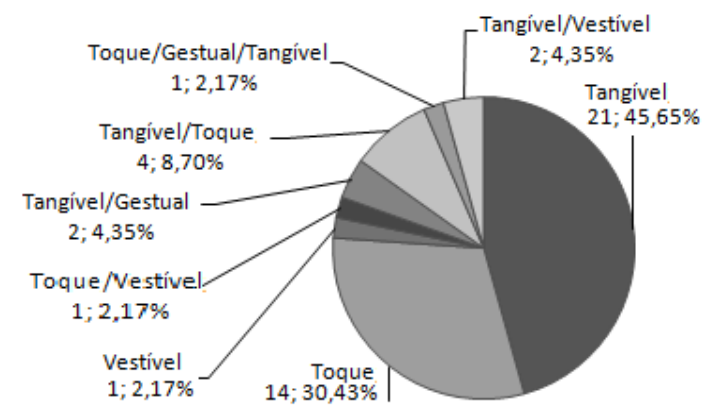

(b)

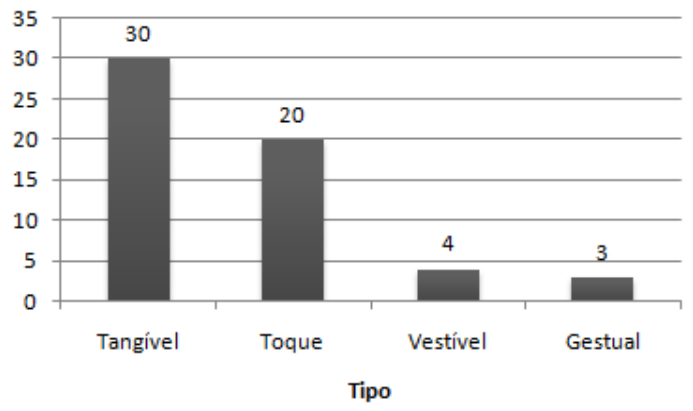

Figura 2 - Tipos de interação/interface utilizados nas ferramentas

\footnotetext{
${ }^{6}$ Os países foram identificados com base nos países das instituições dos autores.
} 
A Figura 3 mostra que $24,53 \%$ do total de ferramentas apresentadas nos trabalhos utilizam a tabletop como dispositivo principal e em seguida aparecem o tablet e o smartphone com 13,21\% e 11,32\% de utilização, respectivamente. A fatia "Outros" refere-se aos tipos de dispositivos que são utilizados por somente uma ferramenta, representando 1,89\% cada. O fato de estas ferramentas que admitem tanto interação por toque quanto tangível contemplarem pelo menos $52,83 \%$ dos tipos de dispositivos usados pelas ferramentas (aqui contabilizando também os dispositivos touchscreen, com $3,77 \%$ ) pode ser devido à sua maior popularização, principalmente em relação aos smartphones e aos tablets, bem como a diminuição do custo destes dispositivos.
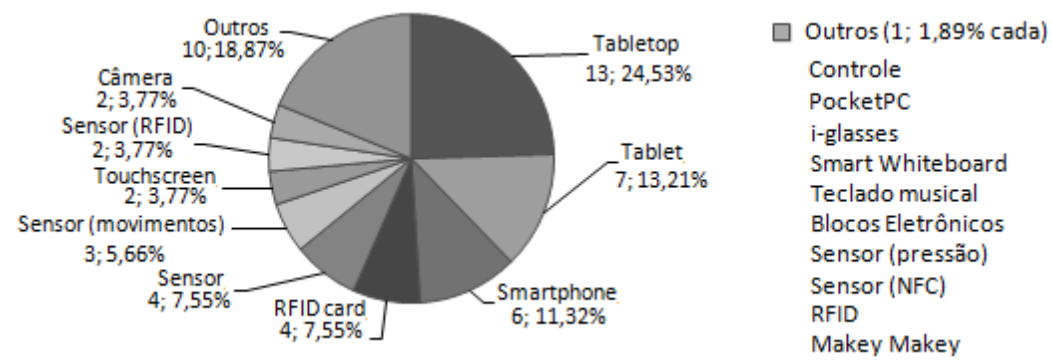

Figura 3 - Tipos de dispositivos utilizados nas ferramentas

A Figura 4, onde cada coluna representa um estudo da RSL, mostra a distribuição, ao longo dos anos, dos dispositivos utilizados pelas ferramentas dos estudos e, é possível perceber um grande aumento da utilização de smartphones e tablets após o ano de 2010.

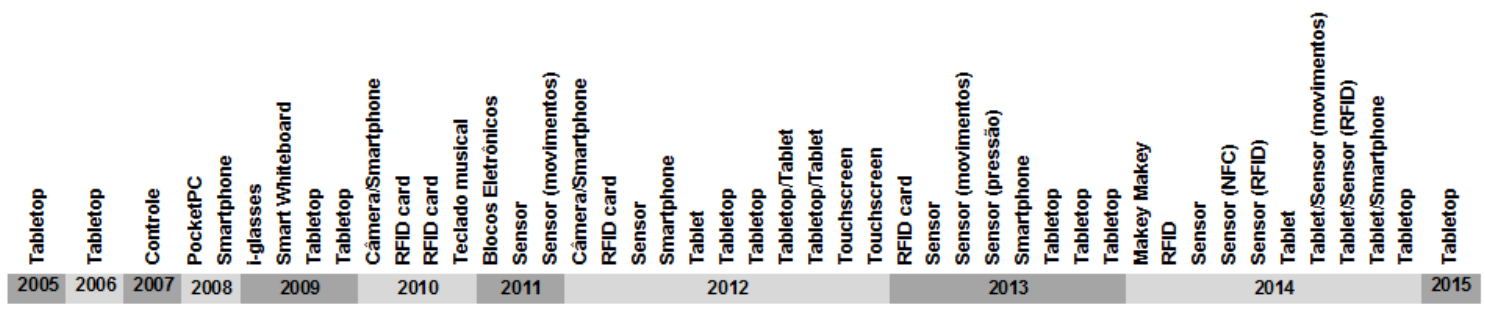

Figura 4 - Distribuição, ao longo dos anos, dos dispositivos utilizados pelas ferramentas apresentadas nos estudos

Por meio da QP2 busca-se conhecer se a solução do estudo foi desenvolvida para um público específico. A Figura 5 apresenta as características do público-alvo das ferramentas. Ferramentas para apoio às pessoas autistas é a característica mais trabalhada, representando $36 \%$ dos estudos. A fatia "Outros" refere-se às características que são focadas por somente uma ferramenta, representando $2,00 \%$ cada.

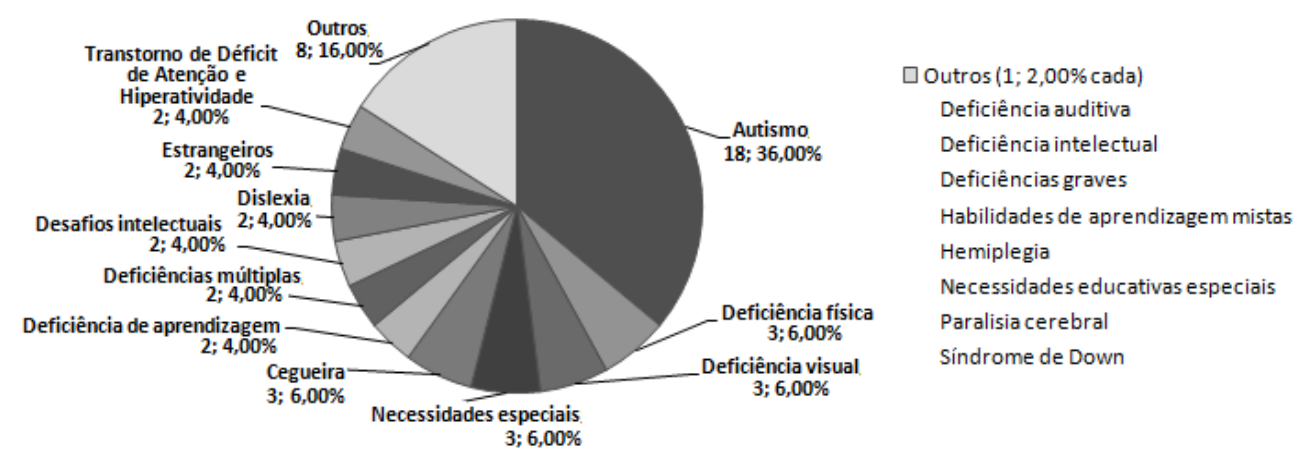

Figura 5 - Características do público-alvo das ferramentas 
A Figura 6 apresenta as características tratadas pelos estudos ao longo dos anos, sendo que alguns deles focam em mais de uma característica. Nesta figura também é possível observar - linha (a) - a porcentagem absoluta de publicações que tratam do autismo em cada ano, além do percentual de estudos sobre autismo relativo à todas as demais características - linha (b) - até o ano em questão. É possível observar que o foco no autismo vem diminuindo desde o ano de 2012, mas ainda é muito representativo em relação às demais característica tratadas pelos estudos.

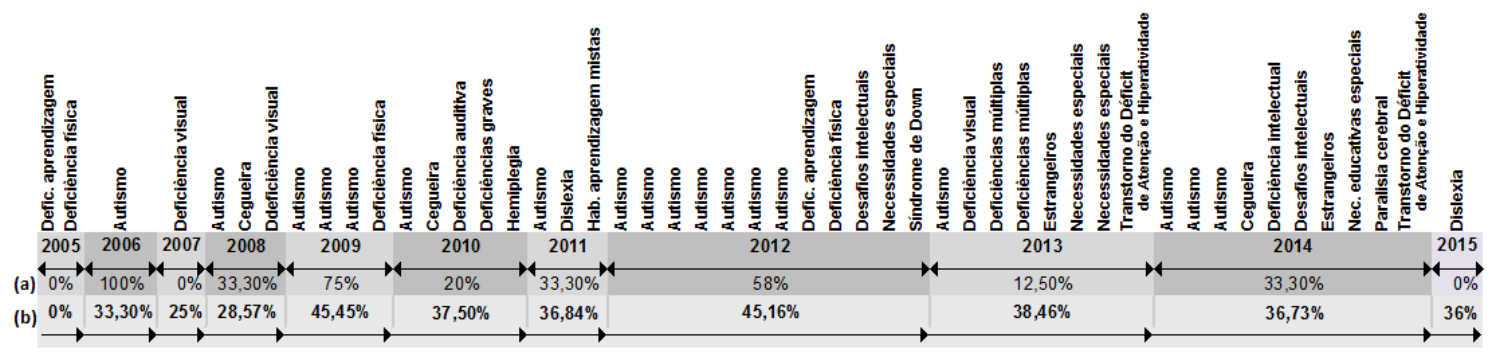

Figura 6 - Distribuição ao longo dos anos das características-alvo das ferramentas e porcentagens de estudos sobre autismo

A Figura 7 apresenta os objetivos a serem alcançados por meio da utilização das ferramentas propostas, ressaltando que alguns estudos visam mais de um objetivo simultaneamente. A aprendizagem é o principal foco $(16,67 \%)$, seguida de interação social e habilidade social, com $13,89 \%$ e $12,50 \%$, respectivamente. A fatia "Outros" refere-se aos objetivos que são trabalhados por somente uma ferramenta, representando $1,39 \%$ cada. Como a maioria dos estudos obtidos nesta RSL é voltada para autistas, era mesmo esperado que aprendizagem, interação social e habilidade social fossem os objetivos mais focados pelos estudos, uma vez que trabalham alterações funcionais do autista [Blasco et al. 2009].
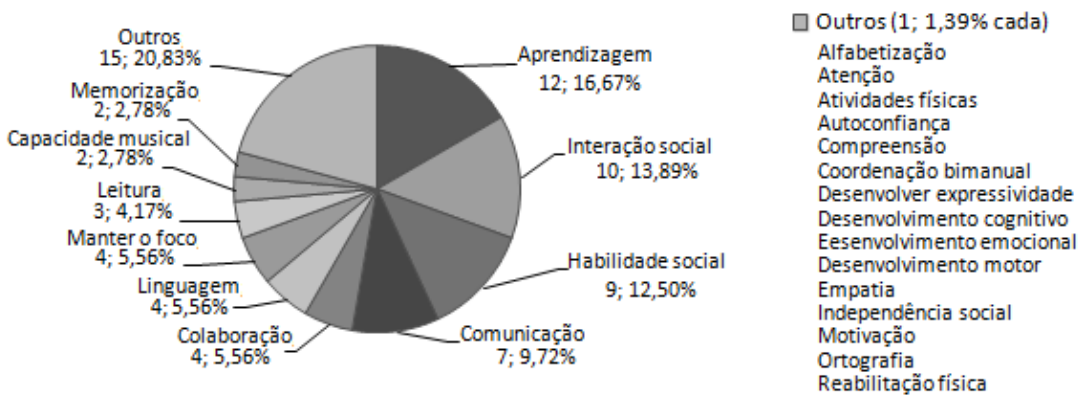

Figura 7 - Objetivos a serem trabalhados por meio das ferramentas

A QP3 refere-se à maneira como as ferramentas foram testadas e com quem foram testadas, quando testadas. A Figura 8.a mostra que quatro das ferramentas propostas pelos pesquisadores não tinham sido ainda testadas quando o trabalho foi publicado. Daquelas que foram testadas, mais de $71 \%$ foram testadas em escolas (Figura 8.b), ambiente no qual as ferramentas seriam mais utilizadas quando em produção. Das cinco ferramentas $(11,90 \%)$ que foram testadas em laboratório da universidade, três $(7,14 \%)$ foram testadas somente no laboratório, sendo que as outras duas $(4,76 \%)$ também foram testadas em escolas.

Em relação aos tipos de avaliação utilizados nos testes (onde avaliação refere-se à forma utilizada pelos pesquisadores para determinar se a ferramenta que está sendo testada atinge o resultado esperado), a "observação" foi o método mais utilizado (Figura 
9). Um dos estudos utilizou a Escala "Social Interaction Observation" (SIO) de "Friendship Observation Scale" (FOS), que consiste em um sistema de codificação interacional projetado para avaliar mudanças minuto a minuto e usado para avaliar o nível de interação social, conversação, cooperação e comportamentos autistas. Outro estudo utilizou "Early Social-Communication Scales" para medição estruturada de habilidades de comunicação não-verbal.

(a)

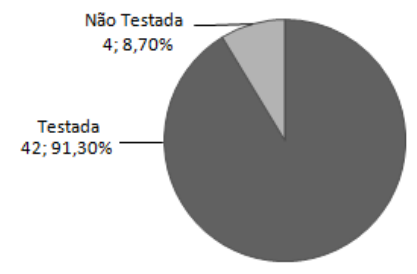

Figura 8 - (a) Teste da ferramenta; (b) Local do teste da ferramenta (b)

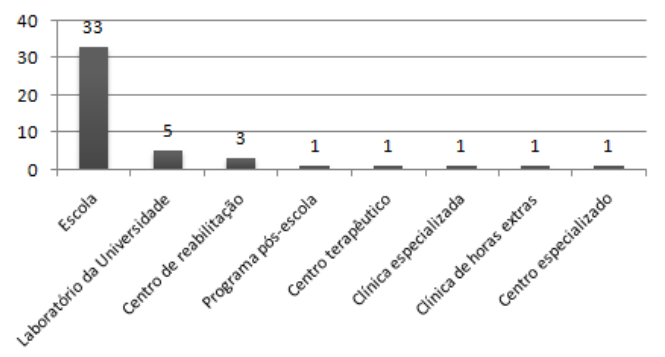

Figura $8-(a)$ Teste da ferramenta; (b) Local do teste da ferramenta

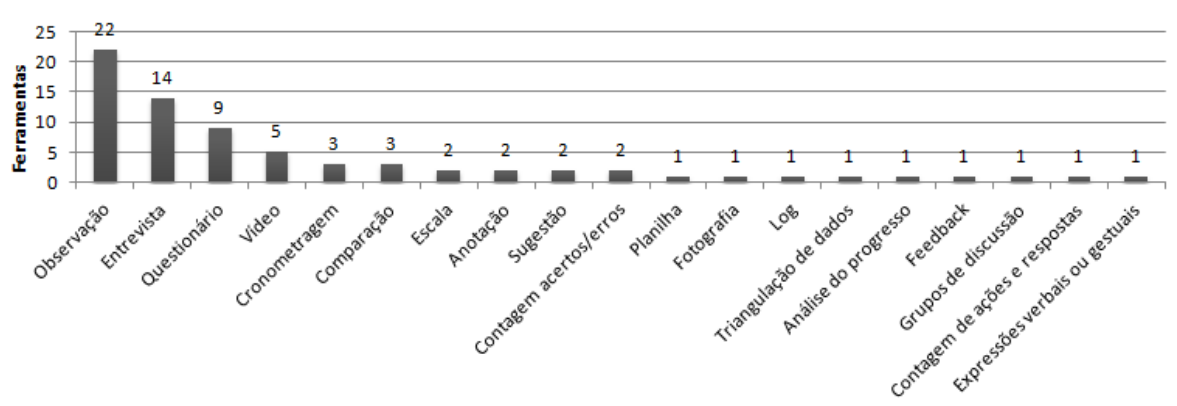

Figura 9 - Tipos de avaliação apresentados nos estudos

Outros tipos de medidas também foram utilizados nas avaliações, tais como medida do padrão de atividade física da criança, erros e acertos, além de comparações entre realização da tarefa sem e com o auxílio da ferramenta ou por meio de interações via mouse. Outro estudo utilizou arquivos de $l o g$, criados automaticamente pela ferramenta, que continham todos os eventos ocorridos durante a sessão, tais como manipulações com objetos físicos e interações com objetos virtuais. De posse dos registros do log, era possível para os pesquisadores fazer uma análise quantitativa das tarefas realizadas. Estudos também utilizaram vídeos e fotografias que serviram para verificar expressões dos usuários e a interação entre eles, bem como a maneira como a ferramenta e os dispositivos envolvidos eram manipulados, podendo assim, identificar problemas de usabilidade, dificuldade de execução de gestos ou execução das tarefas relacionadas à ferramenta, por exemplo. Dois estudos também receberam sugestões de professores ou usuários para melhorias das ferramentas.

\subsection{Discussão}

Os resultados deste estudo apontaram que as tecnologias contemporâneas têm sido utilizadas na concepção de ferramentas para ambientes escolares inclusivos.

Foi possível observar que as tecnologias que utilizam interfaces tangíveis e interações por toque predominam nos estudos, que também apresentam tabletop, tablet 
e smartphones como os tipos de dispositivos mais utilizados. Além disso, as tecnologias vestíveis aparecem em apenas quatro estudos $(8,69 \%)$ que utilizam como dispositivos principais i-glasses, câmera, leitor RFID e sensores em Arduino. A interface gestual também é pouco trabalhada pelos estudos (apenas três estudos). A utilização de ferramentas que utilizam interações por toque ou gestuais pela maioria das pessoas, dentro dos princípios do design universal, entretanto, ainda merece nova investigação.

Foi possível observar também que grande parte dos estudos objetiva a melhoria de condições apresentadas por autistas, mas que este foco no autista tem mudado desde o ano de 2013, onde os pesquisadores passaram a trabalhar com outras deficiências que podem ser apoiadas por recursos tecnológicos, tais como deficiências e desafios intelectuais, paralisia cerebral e Transtorno de Déficit de Atenção e Hiperatividade.

Em relação aos testes, 33 ferramentas (mais de $71 \%$ das ferramentas propostas) foram testadas no ambiente escolar inclusivo, geralmente com o público-alvo da ferramenta. Os estudos levantados, em sua grande maioria $(78,57 \%)$, avaliam as ferramentas propostas em contextos reais (escolas). Pesquisadores de 25 estudos $(54,35 \%)$ também descreveram sobre a fase de concepção da ferramenta, consistindo em entrevistas (9 estudos), observações (11 estudos), revisão da literatura (2 estudos), design participativo (com professores, crianças, terapeutas, especialistas; 6 estudos), além de análise de vídeos e material utilizado por especialistas nas sessões, brainstorm com professores, recomendações de especialistas e discussões (1 estudo cada). Esta concepção da ferramenta baseada em conhecimento do ambiente no qual será utilizada facilita potencialmente seu uso.

Dos três trabalhos desta RSL com autoria de pesquisadores brasileiros, dois fizeram testes com usuários e todos são para utilização individual. Ainda, todos são voltados para interação por toque, ao contrário dos apresentados por pesquisadores estrangeiros, onde a interface tangível prevalece.

\section{Conclusão}

O papel das TICs, como coadjuvantes na promoção da inclusão, aprimorando a utilização dos materiais pedagógicos em ambiente escolares e servindo como mediador no processo de ensino-aprendizagem tem sido reconhecido. Porém pouco se conhece da literatura sobre o potencial das tecnologias contemporâneas tangíveis e vestíveis como recursos para ambientes escolares inclusivos. Com o intuito de conhecer e avaliar tal cenário, este trabalho apresentou uma revisão sistemática da literatura no tema, contemplando os últimos 15 anos de publicações disponíveis nos principais veículos acadêmicos nacionais e internacionais da área de Informática e Educação. No total, 46 publicações provenientes de quatro continentes foram analisadas, após criteriosa seleção realizada de acordo com a metodologia Prisma.

Apesar de a tecnologia vestível ser parte da pergunta de pesquisa, pouco se encontrou sobre o seu uso em ambientes escolares inclusivos. Em síntese, com base nos dados extraídos, a interface tangível e a interação por toque são os tipos de interação mais presentes nos estudos; ainda, quatro estudos $(8,69 \%)$ utilizaram tecnologias vestíveis e três $(6,52 \%)$ utilizaram interação gestual. Em relação ao cenário brasileiro presente nos resultados deste estudo, apesar de haver muitos trabalhos sobre educação inclusiva nas conferências nacionais consultadas, a busca nestas conferências por trabalhos que utilizam tecnologias contemporâneas retornou somente três estudos (um 
utilizando tecnologia tangível e dois com interação por toque). Destes três estudos, dois tinham como audiência a criança com autismo e o terceiro focava em deficiência física e de aprendizagem.

Os resultados deste estudo também mostram que tecnologias vestíveis e gestuais são ainda pouco exploradas, tanto no cenário nacional quanto no internacional, sugerindo um campo aberto de pesquisa. Estender o alcance das tecnologias tangíveis e vestíveis a outros tipos de deficiência também parece um caminho natural de pesquisa que, conforme mostraram os resultados, se concentram bastante no autismo.

Finalmente, os resultados sugerem, ainda, que o design universal de ambientes baseados nessas tecnologias ainda se apresenta como campo de investigação em aberto, com potencial de contribuição aos ambientes inclusivos. Como sugestão de trabalhos futuros, pode-se ampliar esta SRL para conhecer quais tecnologias estão sendo utilizadas com determinados tipos de deficiências.

\section{Agradecimentos}

Este trabalho teve apoio do CNPq (\#308618/2014-9), do Instituto de Computação/Unicamp e da Universidade Federal de Itajubá.

\section{Referências}

Araújo, A. L. S. O, Brito R. R., Silva, A. P. (2013) "Softwares Para Educação Inclusiva: Uma Revisão Sistemática no Contexto de SBIE E WIE.” In Congresso Brasileiro de Informática Na Educação, 507-15.

Blasco, S., Cerro, P., Elena, M., J. D. Uceda. (2009) "Autism and Technology: An Approach to New Technology-Based Therapeutic Tools.” In IFMBE Proc. 340-43.

Galvão Filho, T. A., Damasceno, L. L. (2008) “Tecnologia Assistiva Em Ambiente Computacional: Recursos Para a Autonomia E Inclusão Sócio-Digital Da Pessoa Com Deficiência." In Tecnologias Assitivas Nas Escolas:Recursos Básicos de Acessibilidade Sócio-Digital Para Pessoas Com Deficiência, 25-38. São Paulo: Instituto de Tecnologia Social (ITS Brasil).

Khowaja, K., Salim, S. S. (2013) "A Systematic Review of Strategies and ComputerBased Intervention (CBI) for Reading Comprehension of Children with Autism." In Research in Autism Spectrum Disorders, 1111-21. Elsevier Ltd.

Moher, D., Liberati, A., Tetzlaff, J., Altman, D. G., Prisma Group. (2009) "Preferred Reporting Items for Systematic Reviews and Meta-Analyses: The PRISMA Statement.” In Physical Therapy, 89:873-80.

Ropoli, E. A., Mantoan, M. T. E., Santos, M. T. C. T., Machado, R. (2010) A Escola Comum Inclusiva. Brasília: Ministério da Educaçao.

Sheu, F. R., Chen N. S. (2014) "Taking a Signal: A Review of Gesture-Based Computing Research in Education." Computers and Education 78: 268-77.

Ullmer, B., Ishii, H. (2000) "Emerging Frameworks for Tangible User Interfaces." In IBM Systems Journal, 39: 915-31.

As referências dos estudos da RSL encontram-se em http://www.ic.unicamp.br/ $\sim$ ra120437/SBIE2015. 